Modeling, Identification and Control, Vol. 34, No. 1, 2013, pp. 11-18, ISSN 1890-1328

\title{
Control of Spacecraft Formation with Disturbance Rejection and Exponential Gains
}

\author{
R. Schlanbusch R. Kristiansen \\ Department of Technology, Narvik University College, 8505 Narvik, Norway. E-mail: \{runsch, rayk\}@hin.no
}

\begin{abstract}
We address the problem of state feedback translational motion control of a spacecraft formation through a modified sliding surface controller using variable gains and $I^{2}$ action for disturbance rejection. The exponential varying gains ensure faster convergence of the state trajectories during attitude maneuver while keeping the gains small (and the system less stiff) for station keeping. Integral action is introduced for rejection of disturbances with a constant nonzero mean such as aerodynamic drag. A direct consequence is a drop in energy consumption when affected by sensor noise and a decrease in size of the error states residual when operating close to the equilibrium point. A large number of simulation results are presented to show the control performance.
\end{abstract}

Keywords: Spacecraft Formation, Translational Control, Disturbance Rejection, Nonlinear Gains

\section{Introduction}

In recent years, formation flying has become an increasingly popular subject of study introduced as a new method of performing space operations. By replacing large and complex spacecraft with an array of simpler micro-satellites brings out new possibilities and opportunities of cost, redundancy and improved resolution aspects of onboard payload. Spacecraft formation flying can be defined as a set of spacecraft moving together with control laws inherently coupled trough dynamic states. This coupling can be in translational and/or rotational degrees of freedom and in position and/or velocity Scharf et al. (2004). The main challenge for these types of space missions is the increased requirement of synchronization between leader and follower spacecraft, and robust and reliable control of relative positions is necessary to make the spacecraft cooperate to gain the possible advantages made feasible by spacecraft formations. At this end, behaviors like reconfiguration, formation keeping and collision avoidance are essential for a spacecraft formation to fulfill its desired mission.
Because of the great interest in spacecraft formation flying, modeling and control has received a lot of attention - cf. Kristiansen (2008) and references therein. Basically there are two different approaches for modeling spacecraft formations: Cartesian coordinates and orbital elements. The orbital element method is often used to design formations concerning low fuel expenditure because of the relationship towards natural orbits, while Cartesian models often are used where an orbit with fixed dimensions are studied. The simplest model of relative motion between two spacecraft is linear and known as the Hill Hill (1878) or Clohessy-Wiltshire equations Clohessy and Wiltshire (1960). These linear models are based on assumptions of circular orbits, no orbital perturbations and small relative distance between spacecraft compared with the distance from the formation to the center of the Earth. Nonlinear models as presented in e.g. McInnes (1995); Wang and Hadaegh (1996) and later in (Schaub and Junkins, 2003, Ch. 14), Yan et al. (2000b); Ploen et al. (2004) and Kristiansen (2008) were derived for arbitrary orbital eccentricity and with added terms for orbital perturbations, which are utilized in this paper. 
Numerous different approaches have been proposed for the leader-follower control problem $-c f$. Scharf et al. (2004) including linear feedback control Yan et al. (2000a) linear quadratic optimal control Kapila et al. (1999) and model predictive control Manikonda et al. (1999) to name a few. Many tracking controllers are based on the sliding surface Slotine and Li (1987) and the PD+ Paden and Panja (1988) controllers which are celebrated controllers for robot manipulators - $f$. Kelly et al. (2005) on tracking control of robot manipulators. In Kristiansen and Nicklasson (2009) a thorough review is presented on these types of tracking controllers adapted for spacecraft formation. Variable gains have been utilized on different applications such as the inverted 3D pendulum problem Chaturvedi et al. (2009) and spacecraft attitude control Schlanbusch et al. (2010) where in the latter it was shown that by using exponential gains the trajectories of the closed-loop system converged faster than using static gains, while keeping the gains small for station keeping, reducing the sensibility to measurement noise.

In this paper the results of Schlanbusch et al. (2010) are extended in two different directions and adapted for translational control of spacecraft formations. First, the weighting of the exponential gains are considered for the error of each axis as a state dependent diagonal matrix instead of the state dependent scalar; second, $I^{2}$ action is introduced ( $c f$. Ortega et al. (1995) for output feedback $\mathrm{PI}^{2} \mathrm{D}$ regulator for robot manipulators) to reduce the residual of the physical state vector caused by unknown disturbances. Numerous simulation results are presented comparing performance between the derived controller, a regular sliding surface controller and the strategy presented in Schlanbusch et al. (2010).

The rest of the paper is organized as follows: preliminaries are presented in Section 2, controller design in Section 3, simulation results in Section 4 and wrapping up with conclusions in Section 5.

\section{Modelling}

In the following, the time derivative of a vector $\mathbf{x}$ is denoted by $\dot{\mathbf{x}}$, i.e. $\dot{\mathbf{x}}=d \mathbf{x} / d t$, and moreover, $\ddot{\mathbf{x}}=d^{2} \mathbf{x} / d t^{2}$. The Euclidian norm of a vector and the induced $\mathcal{L}_{2}$ norm of a matrix is denoted by $\|\cdot\|$, and $\mathbb{R}_{+}$as the set of all positive numbers. The cross-product operator is denoted $\mathbf{S}(\cdot)$, such that $\mathbf{S}(\mathbf{x}) \mathbf{y}=\mathbf{x} \times \mathbf{y}$. Reference frames are denoted by $\mathcal{F}^{(\cdot)}$, and in particular, the standard Earth-Centered Inertial (ECI) frame is denoted $\mathcal{F}^{i}$. The angular velocity of $\mathcal{F}^{a}$ relative to $\mathcal{F}^{b}$, referenced in $\mathcal{F}^{c}$ is denoted by $\boldsymbol{\omega}_{b, a}^{c}$. Matrices representing rotation or coordinate transformation from $\mathcal{F}^{a}$ to $\mathcal{F}^{b}$ are denoted $\mathbf{R}_{a}^{b}$. When the context is sufficiently ex-

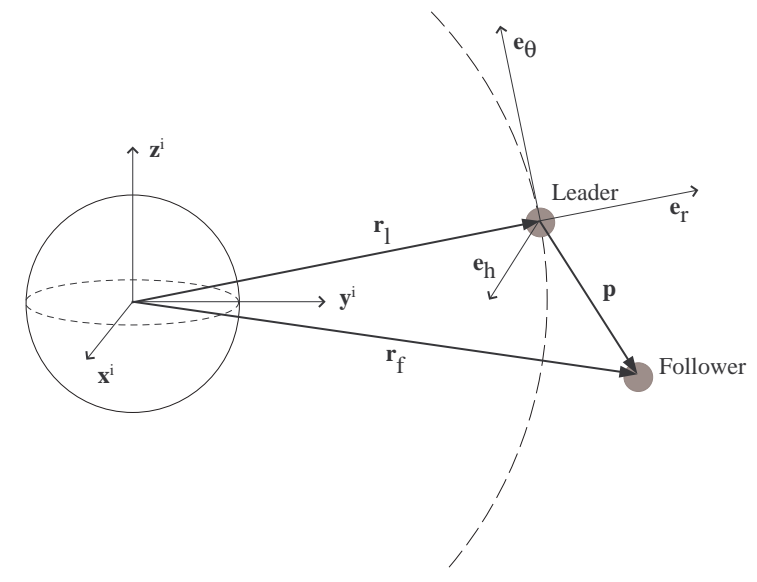

Figure 1: Reference coordinate frames.

plicit, arguments of a function, vector or matrix may be omitted.

\subsection{Cartesian coordinate frames}

The coordinate reference frames used throughout the paper are shown in Figure 1, and defined as follows:

Leader orbit reference frame: The leader orbit frame, denoted $\mathcal{F}^{l}$, has its origin located in the center of mass of the leader spacecraft. The $\mathbf{e}_{r}$ axis in the frame coincide with the vector $\mathbf{r}_{l} \in \mathbb{R}^{3}$ from the center of the Earth to the spacecraft, and the $\mathbf{e}_{h}$ axis is parallel to the orbital angular momentum vector, pointing in the orbit normal direction. The $\mathbf{e}_{\theta}$ axis completes the right-handed orthonormal frame. More rigorously, the basis vectors of the frame are defined as

$$
\mathbf{e}_{r}:=\frac{\mathbf{r}_{l}}{r_{l}}, \quad \mathbf{e}_{\theta}:=\mathbf{S}\left(\mathbf{e}_{h}\right) \mathbf{e}_{r} \quad \text { and } \quad \mathbf{e}_{h}:=\frac{\mathbf{h}}{h},
$$

where $\mathbf{h}=\mathbf{S}\left(\mathbf{r}_{l}\right) \dot{\mathbf{r}}_{l}$ is the angular momentum vector of the orbit, $h=\|\mathbf{h}\|$ and $r_{l}=\left\|\mathbf{r}_{l}\right\|$.

Follower orbit reference frame: This frame has its origin in the center of mass of the follower spacecraft, and is denoted $\mathcal{F}^{f}$. The vector pointing from the center of the Earth to the frame origin is denoted $\mathbf{r}_{f} \in \mathbb{R}^{3}$. The frame is specified by a relative orbit position vector $\mathbf{p}=[x, y, z]^{\top}$ expressed in $\mathcal{F}^{l}$ components, and its unit vectors align with the basis vectors of $\mathcal{F}^{l}$. Accordingly,

$$
\mathbf{p}=\mathbf{R}_{i}^{l}\left(\mathbf{r}_{f}-\mathbf{r}_{l}\right)=x \mathbf{e}_{r}+y \mathbf{e}_{\theta}+z \mathbf{e}_{h} \Rightarrow \mathbf{r}_{f}=\mathbf{R}_{l}^{i} \mathbf{p}+\mathbf{r}_{l} .
$$

\subsection{Formation dynamics}

The relative orientation of reference frames may be represented by a rotation matrix $\mathbf{R} \in S O(3)=\{\mathbf{R} \in$ $\left.\mathbb{R}^{3 \times 3}: \mathbf{R}^{\top} \mathbf{R}=\mathbf{I}, \operatorname{det}(\mathbf{R})=1\right\}$ which is the special 
orthogonal group of order three, and its time derivative can according to (Egeland and Gravdahl, 2002, p. 240) be written as

$$
\dot{\mathbf{R}}_{b}^{a}=\mathbf{S}\left(\boldsymbol{\omega}_{a, b}^{a}\right) \mathbf{R}_{b}^{a}=\mathbf{R}_{b}^{a} \mathbf{S}\left(\boldsymbol{\omega}_{a, b}^{b}\right)
$$

The fundamental differential equation of the two-body problem can be expressed as (Battin, 1999, Ch. 3)

$$
\ddot{\mathbf{r}}_{s}=-\frac{\mu}{r_{s}^{3}} \mathbf{r}_{s}+\frac{\mathbf{f}_{d s}}{m_{s}}+\frac{\mathbf{f}_{a s}}{m_{s}},
$$

where $\mathbf{f}_{d s} \in \mathbb{R}^{3}$ is the perturbation term due to external effects, $\mathbf{f}_{a s} \in \mathbb{R}^{3}$ is the actuator force and $m_{s}$ is the mass of the spacecraft. Subscript $s$ denotes the spacecraft in question, so $s=l, f$ for the leader and follower spacecraft respectively. The spacecraft masses are assumed to be small relative to the mass of the Earth $M_{e}$, so $\mu \approx G M_{e}$, where $G$ is the gravitational constant. According to (2) the relative position between the leader and follower spacecraft may be expressed as

$$
\mathbf{R}_{l}^{i} \mathbf{p}=\mathbf{r}_{f}-\mathbf{r}_{l}
$$

and by differentiating twice

$$
\begin{aligned}
\mathbf{R}_{l}^{i} \ddot{\mathbf{p}} & +2 \mathbf{R}_{l}^{i} \mathbf{S}\left(\boldsymbol{\omega}_{i, l}^{l}\right) \dot{\mathbf{p}} \\
& +\mathbf{R}_{l}^{i}\left(\mathbf{S}^{2}\left(\boldsymbol{\omega}_{i, l}^{l}\right)+\mathbf{S}\left(\dot{\boldsymbol{\omega}}_{i, l}^{l}\right)\right) \mathbf{p}=\ddot{\mathbf{r}}_{f}-\ddot{\mathbf{r}}_{l} .
\end{aligned}
$$

By inserting (4), the right hand side of (6) may be written as

$$
\ddot{\mathbf{r}}_{f}-\ddot{\mathbf{r}}_{l}=-\frac{\mu}{r_{f}^{3}} \mathbf{r}_{f}+\frac{\mathbf{f}_{d f}}{m_{f}}+\frac{\mathbf{f}_{a f}}{m_{f}}+\frac{\mu}{r_{l}^{3}} \mathbf{r}_{l}-\frac{\mathbf{f}_{d l}}{m_{l}}-\frac{\mathbf{f}_{a l}}{m_{l}},
$$

and by inserting (2) into (7), it is found that

$$
\begin{aligned}
m_{f}\left(\ddot{\mathbf{r}}_{f}-\ddot{\mathbf{r}}_{l}\right)= & -m_{f} \mu\left[\left(\frac{1}{r_{f}^{3}}-\frac{1}{r_{l}^{3}}\right) \mathbf{r}_{\mathbf{l}}+\frac{\mathbf{R}_{l}^{i} \mathbf{p}}{r_{f}^{3}}\right] \\
& +\mathbf{f}_{a f}+\mathbf{f}_{d f}-\frac{m_{f}}{m_{l}}\left(\mathbf{f}_{a l}+\mathbf{f}_{d l}\right) .
\end{aligned}
$$

Moreover, by inserting (8) into (6), and rearranging the terms

$$
\begin{aligned}
m_{f} \ddot{\mathbf{p}}+\mathbf{C}_{t}\left(\boldsymbol{\omega}_{i, l}^{l}\right) \dot{\mathbf{p}} & +\mathbf{D}_{t}\left(\dot{\boldsymbol{\omega}}_{i, l}^{l}, \boldsymbol{\omega}_{i, l}^{l}, r_{f}\right) \mathbf{p} \\
& +\mathbf{n}_{t}\left(\mathbf{r}_{l}, r_{f}\right)=\mathbf{F}_{a}+\mathbf{F}_{d}
\end{aligned}
$$

where

$$
\mathbf{C}_{t}\left(\boldsymbol{\omega}_{i, l}^{l}\right)=2 m_{f} \mathbf{S}\left(\boldsymbol{\omega}_{i, l}^{l}\right)
$$

is a skew-symmetric matrix,

$$
\mathbf{D}_{t}\left(\dot{\boldsymbol{\omega}}_{i, l}^{l}, \boldsymbol{\omega}_{i, l}^{l}, r_{f}\right)=m_{f}\left[\mathbf{S}^{2}\left(\boldsymbol{\omega}_{i, l}^{l}\right)+\mathbf{S}\left(\dot{\boldsymbol{\omega}}_{i, l}^{l}\right)+\frac{\mu}{r_{f}^{3}} \mathbf{I}\right],
$$

may be viewed as a time-varying potential force, and

$$
\mathbf{n}_{t}\left(\mathbf{r}_{l}, r_{f}\right)=\mu m_{f} \mathbf{R}_{i}^{l}\left[\frac{1}{r_{f}^{3}}-\frac{1}{r_{l}^{3}}\right] \mathbf{r}_{l}
$$

is a nonlinear term. The composite perturbation force $\mathbf{F}_{d}$ and the composite relative control force $\mathbf{F}_{a}$ are given respectively by

$$
\mathbf{F}_{d}=\mathbf{R}_{i}^{l}\left(\mathbf{f}_{d f}-\frac{m_{f}}{m_{l}} \mathbf{f}_{d l}\right), \mathbf{F}_{a}=\mathbf{R}_{i}^{l}\left(\mathbf{f}_{a f}-\frac{m_{f}}{m_{l}} \mathbf{f}_{a l}\right) .
$$

Note that all forces $\mathbf{f}$ are given in inertial frame. If the forces are computed in another frame, the rotation matrix should be replaced accordingly. The orbit angular velocity and angular acceleration can be written as $\boldsymbol{\omega}_{i, l}^{i}=\mathbf{S}\left(\mathbf{r}_{l}\right) \mathbf{v}_{l} / \mathbf{r}_{l}^{\top} \mathbf{r}_{l}$, and

$$
\dot{\omega}_{i, l}^{i}=\frac{\mathbf{r}_{l}^{\top} \mathbf{r}_{l} \mathbf{S}\left(\mathbf{r}_{l}\right) \mathbf{a}_{l}-2 \mathbf{v}_{l}^{\top} \mathbf{r}_{l} \mathbf{S}\left(\mathbf{r}_{l}^{\top}\right) \mathbf{v}_{l}}{\left(\mathbf{r}_{l}^{\top} \mathbf{r}_{l}\right)^{2}}
$$

respectively. Then $\boldsymbol{\omega}_{i, l}^{l}=\mathbf{R}_{i}^{l} \boldsymbol{\omega}_{i, l}^{i}$ and $\dot{\boldsymbol{\omega}}_{i, l}^{l}=$ $\mathbf{S}\left(\boldsymbol{\omega}_{l, i}^{l}\right) \mathbf{R}_{i}^{l} \boldsymbol{\omega}_{i, l}^{i}+\mathbf{R}_{i}^{l} \dot{\boldsymbol{\omega}}_{i, l}^{i}=-\mathbf{S}\left(\boldsymbol{\omega}_{i, l}^{l}\right) \mathbf{R}_{i}^{l} \boldsymbol{\omega}_{i, l}^{i}+\mathbf{R}_{i}^{l} \dot{\boldsymbol{\omega}}_{i, l}^{i}$.

\section{Control Problem}

For control of the relative translation, a sliding surface controller with $I^{2}$ action and variable gains, inspired by the one derived for trajectory tracking of robot manipulators Slotine and Li (1987), is incorporated. The following assumptions are posed

Assumption 1 It is assumed that the leader spacecraft is perfectly controlled in its orbit, so that $\mathbf{f}_{a l}=$ $-\mathbf{f}_{d l}$, hence $\mathbf{F}_{a}=\mathbf{f}_{a f}$ and $\mathbf{F}_{d}=\mathbf{f}_{d f}$.

Assumption 2 All references functions are continuous and bounded such that $\left\|\ddot{\mathbf{p}}_{d}(t)\right\|<\beta_{\ddot{p}} \in \mathbb{R}_{+}$, $\|\dot{\mathbf{p}}(t)\|<\beta_{\dot{p}} \in \mathbb{R}_{+}$and $\left\|\mathbf{p}_{d}(t)\right\|<\beta_{p} \in \mathbb{R}_{+}$for all $t \geq t_{0}$, and furthermore, the orbital angular velocity of the leader spacecraft is continuous and bounded such that $\left\|\boldsymbol{\omega}_{i, l}^{l}\right\|<\beta_{l} \in \mathbb{R}_{+}$for all $t \geq t_{0}$.

Assumption 3 The perturbation vector can be written as

$$
\mathbf{f}_{d f}=\mathbf{a}+\mathbf{b}(t)
$$

where $\mathbf{a}$ is considered as a constant mean while $\mathbf{b}(t)$ is considered as a higher frequency component, and the latter is assumed upper bounded such that $\|\mathbf{b}(t)\| \leq \beta_{d}$ and $\|\dot{\mathbf{b}}(t)\| \leq \beta_{\dot{d}}$ for all $t \geq t_{0}$.

The assumption given in (15) is reasonable for typical disturbances working on spacecraft such as aerodynamic drag, $J_{2}$ effect caused by uneven mass distribution of the Earth, Solar radiation and third-body 
perturbing forces which all can be seen as state dependent, slow varying and even in some cases constant.

The control goal is to design a tracking control law for the system (9) which is able to track a time varying reference represented by $\ddot{\mathbf{p}}_{d}(t), \dot{\mathbf{p}}_{d}(t)$ and $\mathbf{p}_{d}(t)$ fulfilling $\dot{\mathbf{e}}_{p}(t)=\mathbf{e}_{v}(t)$, where the error functions are defined as $\mathbf{e}_{p}:=\mathbf{p}-\mathbf{p}_{d}=\left[\begin{array}{lll}e_{p x}, & e_{p y}, e_{p z}\end{array}\right]^{\top}$ and $\mathbf{e}_{v}:=\dot{\mathbf{p}}-\dot{\mathbf{p}}_{d}=\left[e_{v x}, e_{v y}, e_{v z}\right]^{\top}$. To this end:

Proposition 1 Under Assumptions 1-3, the solution trajectories of the system (9) in closed-loop with the control law

$$
\begin{aligned}
\mathbf{f}_{a f}= & m_{f} \ddot{\mathbf{p}}_{r}+\mathbf{C}_{t}\left(\boldsymbol{\omega}_{i, l}^{l}\right) \dot{\mathbf{p}}_{r}+\mathbf{D}\left(\dot{\boldsymbol{\omega}}_{i, l}^{l}, \boldsymbol{\omega}_{i, l}^{l}, r_{f}\right) \mathbf{p} \\
& +\mathbf{n}_{t}\left(\mathbf{r}_{l}, r_{f}\right)-\mathbf{K}_{p} \mathbf{e}_{p}-k_{i} \boldsymbol{\zeta}-k_{a} \boldsymbol{\xi}-\mathbf{K}_{d} \mathbf{S} \\
\dot{\boldsymbol{\zeta}}= & \mathbf{e}_{p}, \\
\dot{\boldsymbol{\xi}}= & k_{a} \mathbf{e}_{v}, \\
\mathbf{s}= & {\left[s_{x}, s_{y}, s_{z}\right]^{\top}=\dot{\mathbf{p}}-\dot{\mathbf{p}}_{r}=\mathbf{e}_{v}+\gamma \mathbf{e}_{p}, } \\
\dot{\mathbf{p}}_{r}= & \dot{\mathbf{p}}_{d}-\gamma \mathbf{e}_{p}, \\
\ddot{\mathbf{p}}_{r}= & \ddot{\mathbf{p}}_{d}-\gamma \mathbf{e}_{v},
\end{aligned}
$$

where $\mathbf{K}_{p}=k_{p} \operatorname{diag}\left\{e^{k_{1} e_{p x}^{2}}, \quad e^{k_{1} e_{p y}^{2}}, e^{k_{1} e_{p z}^{2}}\right\}$ and $\mathbf{K}_{d}=k_{d} \operatorname{diag}\left\{e^{k_{2} s_{x}^{2}}, e^{k_{2} s_{y}^{2}}, e^{k_{2} s_{z}^{2}}\right\}$, tuning parameters $k_{p}, k_{i}, k_{a}, k_{d} \in \mathbb{R}_{+}$, and $\gamma=k_{i} / k_{a}^{2}$, is converging uniformly towards the set $\left\{\left(\mathbf{e}_{p}, \mathbf{e}_{v}, \boldsymbol{\zeta}, \boldsymbol{\xi}\right) \in\right.$ $\left.\mathbb{R}^{12}:\left\|\left[\mathbf{e}_{p}, \mathbf{e}_{v}\right]\right\| \leq \delta\right\}$, where $\delta$ is to be defined, for all initial values as $t \rightarrow \infty$.

\section{Proof:}

By inserting the control law (16)-(21) into the dynamics (9) and performing the coordinate transformation

$$
\mathbf{z}=\boldsymbol{\xi}-\frac{1}{k_{a}} \mathbf{a}+\frac{k_{i}}{k_{a}} \boldsymbol{\zeta}
$$

the following closed-loop dynamics are obtained

$$
m_{f} \dot{\mathbf{s}}=-\left(\mathbf{C}_{t} \mathbf{K}_{d}\right) \mathbf{s}-\mathbf{K}_{p} \mathbf{e}_{p}-k_{a} \mathbf{z}+\mathbf{b}(t) .
$$

Defining $\mathbf{x}=\left[\mathbf{e}_{p}^{\top}, \mathbf{s}^{\top}, \mathbf{z}^{\top}\right]^{\top}$, the following Lyapunov Function Candidate (LFC) is proposed

$$
\begin{aligned}
V(\mathbf{x})= & \frac{1}{2}\left(\frac{k_{p}}{k_{1}}\left[e^{k_{1} e_{p x}^{2}}+e^{k_{1} e_{p y}^{2}}+e^{k_{1} e_{p z}^{2}}-3\right]\right. \\
& \left.+\mathbf{s}^{\top} m_{f} \mathbf{s}+\mathbf{z}^{\top} \mathbf{z}\right)
\end{aligned}
$$

which is positive definite and proper, which is shown next by finding functions $\underline{\alpha}(\mathbf{x}), \bar{\alpha}(\mathbf{x}) \in \mathcal{K}_{\infty}$ such that $\underline{\alpha}(\mathbf{x}) \leq V(\mathbf{x}) \leq \bar{\alpha}(\mathbf{x})$. Imposing standard Euclidian norms, the following may be written

$$
V(\mathbf{x}) \leq \frac{1}{2}\left[3 \frac{k_{p}}{k_{1}}\left(e^{k_{1}\|\mathbf{x}\|^{2}}-1\right)+\left(1+m_{f}\right)\|\mathbf{x}\|^{2}\right] .
$$

As typically an exponential function outgrows a squared function thus a constant $c$ is needed such that $e^{c\|\mathbf{x}\|^{2}}-1 \geq\|\mathbf{x}\|^{2}$, which is satisfied for

$$
c \geq \sup _{\mathbf{x} \in \mathbb{R}^{9}} \frac{\ln \left(\|\mathbf{x}\|^{2}+1\right)}{\|\mathbf{x}\|^{2}}=1 .
$$

Hence from (25)

$$
V(\mathbf{x}) \leq \bar{\alpha}(\mathbf{x}):=c_{1}\left(e^{c_{2}\|\mathbf{x}\|^{2}}-1\right)
$$

where $\quad c_{1}:=2 \max \left\{k_{p} / k_{1}, 1+m_{f}\right\}$ and $c_{2} \quad:=$ $\max \left\{k_{1}, 1\right\}$.

Next, a quadratic lower bound on $V$ is found. Recalling that

$$
e^{x}=\sum_{n=0}^{\infty} \frac{x^{n}}{n !} \geq 1+x,
$$

obtaining

$$
\left(e^{k_{1} e_{p x}^{2}}+e^{k_{1} e_{p y}^{2}}+e^{k_{1} e_{p z}^{2}}-3\right) \geq k_{1}\left(e_{p x}^{2}+e_{p y}^{2}+e_{p z}^{2}\right) .
$$

Accordingly from (25)

$$
\underline{\alpha}(\mathbf{x}):=c_{3}\|\mathbf{x}\|^{2},
$$

where $c_{3}=1 / 2 \min \left\{k_{p}, m_{f}, 1\right\}$. Next, the total time derivative of $V$ along the closed-loop trajectories is evaluated, i.e.

$$
\begin{aligned}
\dot{V}(t, \mathbf{x})= & -\mathbf{s}^{\top}\left(\mathbf{C}_{t}+\mathbf{K}_{d}\right) \mathbf{s}-\mathbf{s}^{\top} \mathbf{K}_{p} \mathbf{e}_{p}-\mathbf{e}_{v}^{\top} \mathbf{K}_{p} \mathbf{e}_{p} \\
& -\mathbf{s}^{\top} k_{a} \mathbf{z}+\mathbf{s}^{\top} \mathbf{b}(t)+\mathbf{z}^{\top}\left(k_{a} \mathbf{e}_{v}+\frac{k_{i}}{k_{a}} \mathbf{e}_{p}\right) .
\end{aligned}
$$

By inserting (19) and defining $\gamma:=k_{i} / k_{a}^{2}$, obtaining

$$
\begin{aligned}
\dot{V}(t, \mathbf{x}) & \leq-\mathbf{s}^{\top} k_{d} \mathbf{s}-\mathbf{e}_{p}^{\top} \gamma k_{p} \mathbf{e}_{p}+\mathbf{s}^{\top} \mathbf{b}(t), \\
& \leq-\chi^{\top} \mathbf{p} \boldsymbol{\chi}+\beta_{d}\|\chi\|,
\end{aligned}
$$

where $\boldsymbol{\chi}=\left[\mathbf{e}_{p}^{\top}, \mathbf{s}^{\top}\right]^{\top}$ and $\mathbf{P}=\operatorname{diag}\left\{\gamma k_{p} \mathbf{I}, k_{d} \mathbf{I}\right\}$. By defining $\delta:=\beta_{d} / p_{m}$ where $p_{m}$ is the smallest eigenvalue of $\mathbf{P}$ it is ensured that $\dot{V}(\mathbf{x}) \leq 0$ for all $\|\chi\| \geq \delta$, and thus uniform practical stability of the equilibrium point is obtained, i.e. $V\left(t_{2}\right) \leq V\left(t_{1}\right), \forall t_{2}>t_{1}>t_{0}$ when $\|\chi\| \geq \delta$, and thus it follows that the state $\mathbf{x}$ is bounded. To prove convergence Barbalat's lemma is applied. Note that Barbalat's lemma is imposed in a conservative manner, i.e., to ensure that the physical states converge to the set $\left\{\mathbf{x} \in \mathbb{R}^{9}:\|\chi\| \leq \delta\right\}$ which contains the origin. By differentiation of (31)

$$
\begin{aligned}
\ddot{V}(t, \mathbf{x})= & 2 \mathbf{s}^{\top}\left(\mathbf{C}_{t}+\mathbf{K}_{d}\right) \mathbf{s}+2 \mathbf{s}^{\top} \mathbf{K}_{p} \mathbf{e}_{p}+2 \mathbf{s}^{\top} k_{a} \mathbf{z} \\
& -2 \mathbf{s}^{\top} \mathbf{b}(t)-2 \mathbf{e}_{p}^{\top} k_{p} \mathbf{e}_{v}-\mathbf{s}^{\top}\left(\mathbf{C}_{t}^{\top}+\mathbf{K}_{d}\right) \mathbf{b}(t) \\
& -\mathbf{e}_{p} \mathbf{K}_{p} \mathbf{b}(t)-\mathbf{z}^{\top} k_{a} \mathbf{b}(t)+\mathbf{b}(t)^{\top} \mathbf{b}(t)+\mathbf{s}^{\top} \mathbf{b}(t),
\end{aligned}
$$


where all members are either shown to be bounded or bounded by hypothesis, thus $\ddot{V}(t, \mathbf{x})$ is bounded, which proves that $\dot{V}(t, \mathbf{x})$ is uniformly continuous. Moreover, since $V(\mathbf{x})$ is lower bounded and $\dot{V}(t, \mathbf{x})$ is negative semi-definite for $\|\chi\| \geq \delta$, Barbalat's lemma is imposed to obtain that $\|\chi\| \rightarrow \delta$ as $t \rightarrow \infty$.

Remark 1 The assumption of perfectly control leader and its bounded orbital angular velocity can be relaxed by following the lines of (Grotli, 2010, Ch. 4.2). In the latter paper, a control law for the leader is utilized, leading to a stability proof based on cascaded system including a bound on the leader references similar to Assumption 2 for the follower spacecraft.

Remark 2 By inserting $\mathbf{s}=\mathbf{e}_{v}+\gamma \mathbf{e}_{p}$ and $\gamma=k_{i} / k_{a}^{2}$ into the control law, it can be seen that the proportional term is on the form $-\left(\mathbf{K}_{d} k_{i} / k_{a}^{2}+\mathbf{K}_{p}\right) \mathbf{e}_{p}$ which means that the proportional gain will increase by increasing the gain for the integration of the position error, while decrease with fast integration of the velocity error.

Remark 3 In the control law and simulations it will be assumed that all constants are unknown, but as many of the disturbances for spacecraft can be reasonably well modeled as $\hat{\mathbf{f}}_{d f}^{b}$, this could be added to the overall analysis such that $\tilde{\mathbf{f}}_{d f}^{b}=\mathbf{f}_{d f}^{b}-\hat{\mathbf{f}}_{d f}^{b}=\tilde{\mathbf{a}}+\tilde{\mathbf{b}}(t)$. This strategy could reduce the upper bound such that $\|\tilde{\mathbf{b}}(t)\|<\beta_{\tilde{d}}<\beta_{d}$, based on the quality of the disturbance modeling.

\section{Simulation Results}

In this section simulation results are presented for two different scenarios showing the features of the control law (16). The first simulation results show that the integral action manages to reduce the residual of the practical result, i.e. by comparing (16) with the same controller except with $k_{a}=k_{i}=0$. In the second run of simulations the control law (16)-(21) is compared with an ordinary sliding surface controller as in (Kristiansen, 2008, p. 60) given by

$$
\begin{aligned}
\mathbf{f}_{a f}^{\prime}= & m_{f} \ddot{\mathbf{p}}_{r}+\mathbf{C}_{t}\left(\boldsymbol{\omega}_{i, l}^{l}\right) \dot{\mathbf{p}}_{r}+\mathbf{D}\left(\dot{\boldsymbol{\omega}}_{i, l}^{l}, \boldsymbol{\omega}_{i, l}^{l}, r_{f}\right) \mathbf{p}+\mathbf{n}_{t}\left(\mathbf{r}_{l}, r_{f}\right) \\
& -k_{p} \mathbf{e}_{p}-k_{d} \mathbf{S}
\end{aligned}
$$

Moreover, a comparison with a sliding surface version based on the concept of Schlanbusch et al. (2010) given by

$$
\begin{aligned}
\mathbf{f}_{a f}^{\prime \prime}= & m_{f} \ddot{\mathbf{p}}_{r}+\mathbf{C}_{t}\left(\boldsymbol{\omega}_{i, l}^{l}\right) \dot{\mathbf{p}}_{r}+\mathbf{D}\left(\dot{\boldsymbol{\omega}}_{i, l}^{l}, \boldsymbol{\omega}_{i, l}^{l}, r_{f}\right) \mathbf{p}+\mathbf{n}_{t}\left(\mathbf{r}_{l}, r_{f}\right) \\
& -k_{p} e^{k_{1}\left\|\mathbf{e}_{p}\right\|^{2}} \mathbf{e}_{p}-k_{d} e^{k_{2}\|\mathbf{s}\|^{2}} \mathbf{s},
\end{aligned}
$$

is also performed, where the gains are scalar and vary according to the total length of the error vectors instead of the length along each axis separately. Through these comparisons, it is shown that using variable gain matrices reduces the energy consumption without increasing the settling time.

Simulations were performed in Simulink using a fixed step Runge-Kutta ODE3 solver with step size of $0.01 \mathrm{~s}$. The spacecraft orbit was chosen as elliptic with apogee at $750 \mathrm{~km}$ and perigee at $600 \mathrm{~km}$ altitude, inclination of $71^{\circ}$ and the right ascension of the ascending node and argument of perigee at $0^{\circ}$. Measurement noise is introduced as $\sigma \mathbb{B}^{n}=\left\{x \in \mathbb{R}^{n}:\|x\| \leq \sigma\right\}$ and add a suitable amount to the error functions according to $\tilde{\mathbf{e}}_{p}=\mathbf{e}_{p}+1 \times 10^{-3} \mathbb{B}^{3}$ and $\tilde{\mathbf{e}}_{v}=\mathbf{e}_{v}+5 \times 10^{-4} \mathbb{B}^{3}$. Atmospheric drag and uneven mass distribution of the Earth $\left(J_{2}\right)$ were added according to (Kristiansen, 2008, Ch. 3.5) where all perturbations typically are continuous and slowly varying, while atmospheric drag often can be considered close to constant for near circular orbits.

To evaluate and compare the performance of the controllers the following performance functionals are utilized

$$
\begin{gathered}
J_{p}=\int_{t_{0}}^{t_{f}} \mathbf{e}_{p}^{\top} \mathbf{e}_{p} d t, J_{v}=\int_{t_{0}}^{t_{f}} \mathbf{e}_{v}^{\top} \mathbf{e}_{v} d t \\
J_{u}=\int_{t_{0}}^{t_{f}} \mathbf{f}_{a f}^{\top} \mathbf{f}_{a f} d t
\end{gathered}
$$

where $t_{0}$ and $t_{f}$ define the start and end of the simulation window, respectively. The functional $J_{p}$ and $J_{v}$ describe the integral functional error of the position and velocity error, while $J_{u}$ describes the integral of the applied control force.

For our first scenario the initial conditions were chosen as $\mathbf{p}\left(t_{0}\right)=\left[\begin{array}{lll}20, & -80, & 0\end{array}\right]^{\top} \mathrm{m}$ and $\dot{\mathbf{p}}\left(t_{0}\right)=\mathbf{0}$, $\mathbf{p}_{d}=\left[\begin{array}{ll}10,20, & -30\end{array}\right]^{\top} \mathrm{m}$ and $\dot{\mathbf{p}}_{d}=\ddot{\mathbf{p}}_{d}=\mathbf{0}$, with controller parameters $k_{p}=0.1, k_{d}=7, k_{1}=10^{-4}$, $k_{2}=0.01, k_{i}=10^{-4}$ and $k_{a}=\sqrt{10} \times 10^{-1}$. Simulation results for a translational maneuver are shown in Figure 2 and performance functionals presented in Table 1, which both show that by introducing integral action, the system response is slower during maneuver. On the other hand, by looking at Figure 3 it can be seen that the relative distance error decreases by applying $I^{2}$ action, even if the disturbances are time varying.

The results from the second simulation scenario are presented in Table 2 from 10, 000 simulations for translational maneuvers without disturbances and noise using controller gains $k_{p}=0.1, k_{d}=10$ and $\gamma=0.001$ for (33), and in addition $k_{1}=10^{-4}$ and $k_{2}=10^{-2}$ for (16) and (34). Note that $k_{a}=k_{i}=0$ to study the effect of gain matrices compared to variable scalar gains and static gains. Random initial values for the initial relative position and velocity errors were utilized, with standard deviation of 50 and five, respectively. This was done to show that using exponential gains where 
Table 1: Values of performance functionals for translational maneuver and station keeping

\begin{tabular}{lccc} 
& $J_{p}$ & $J_{v}$ & $J_{u}$ \\
\hline Ctrl. $(16), t \in[0,500]$ & $3.43 \times 10^{5}$ & 133 & 5803 \\
Ctrl. $(16), k_{a}=k_{i}=0, t \in[0,500]$ & $2.40 \times 10^{5}$ & 159 & 5551 \\
Ctrl. $(16), t \in[3000,10000]$ & 7.765 & $1.1 \times 10^{-4}$ & 1.59 \\
Ctrl. $(16), k_{a}=k_{i}=0, t \in[3000,10000]$ & 46.4 & $4.9 \times 10^{-4}$ & 1.60
\end{tabular}
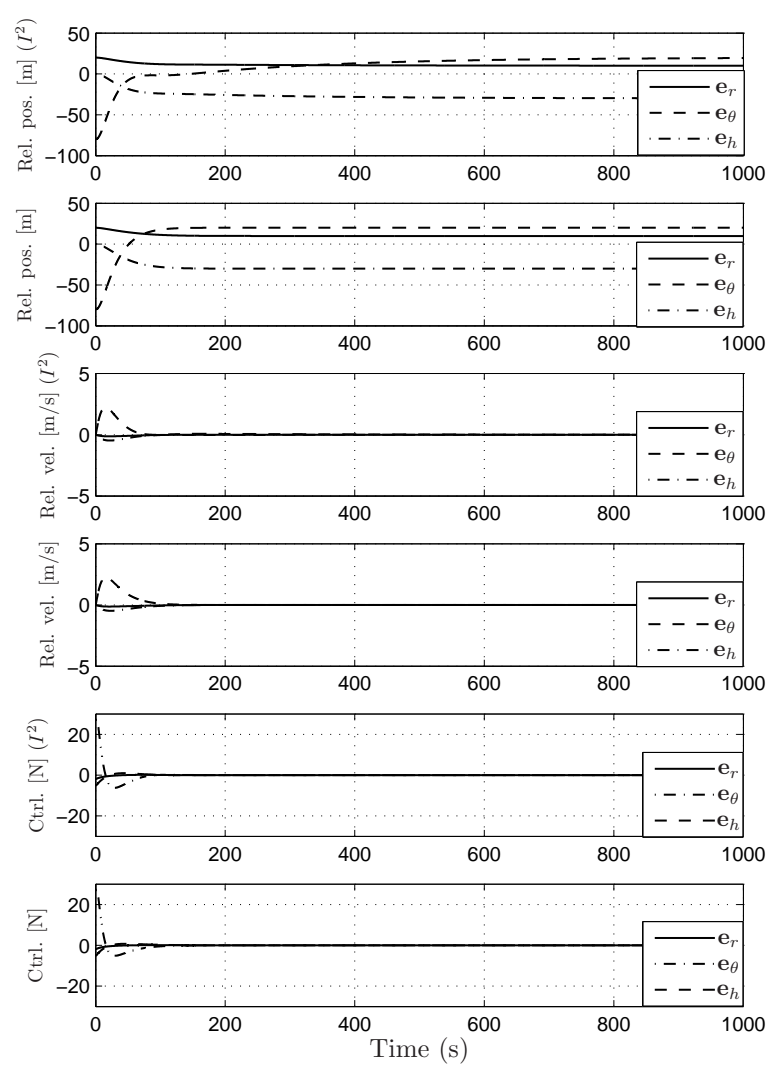

Figure 2: Relative position, relative velocity and control force for translational maneuver by sliding surface control with exponential gains with (plots 1, 3 and 5) and without (plots $2,4,6) I^{2}$ action.

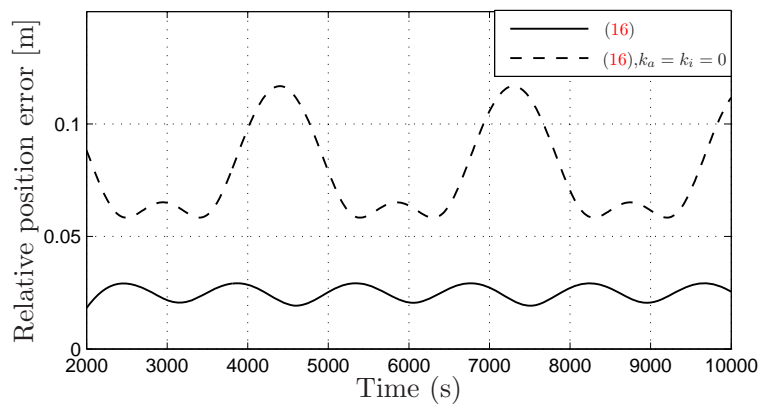

Figure 3: Relative distance error by sliding surface control with exponential gains with and without $I^{2}$ action during station keeping.

Table 2: Average value of performance functionals for rigid-body over 10,000 simulations

\begin{tabular}{lccc} 
& $J_{p}$ & $J_{v}$ & $J_{u}$ \\
\hline Ctrl. (16) & $1.29 \times 10^{6}$ & $1.13 \times 10^{3}$ & $3.76 \times 10^{4}$ \\
Ctrl. (33) & $2.01 \times 10^{6}$ & 884 & $2.67 \times 10^{4}$ \\
Ctrl. (34) & $1.25 \times 10^{6}$ & $1.37 \times 10^{3}$ & $5.48 \times 10^{4}$
\end{tabular}

each axis is weighted differently based on its own error, makes the system work faster than when using static gains, while consuming less energy compared to a control law utilizing exponential gains based on the total length (scalar) of the error vector.

As shown in Schlanbusch et al. (2010) it can be seen that by using variable gains, the gains are large when the solution trajectory is far away from the equilibrium point and goes towards $k_{p}$ and $k_{d}$ when the errors converge towards zero. Thus in general, smaller $k_{p}$ and $k_{d}$ is utilized in (16) compared to (33) for similar performance during transition. This means that the system will be less stiff when working close to the equilibrium and thus less affected by sensor noise. For a spacecraft it follows that less energy is consumed during station keeping which often is a large part of the operation time. As for practical purposes it seems reasonable to introduce a supervisor which will modify the gains during the different stages of spacecraft operation by e.g. using (16) for station keeping while setting $k_{i}=k_{a}=0$ during maneuvers. 


\section{Conclusions}

In this paper, an improvement of the the existing sliding surface control law is presented by introducing variable proportional and derivative gains for control of a leader-follower spacecraft formation, and included integral action to reduce the residual of the error norm caused by unknown disturbances. It was shown that the solution trajectories of the closed-loop system converged uniformly to a set which could be arbitrarily diminished by increasing the controller gains for all initial values under the effect of disturbances. Simulation results showed that the integral action was capable of diminishing the residual caused by typical disturbances encountered by spacecraft, and also that the proposed controller in general works faster than the ordinary sliding surface controller with an increase in power consumption or can work equally fast but be less sensitive to sensor noise during station keeping.

\section{References}

Battin, R. H. An Introduction to the Mathematics and Methods of Astrodynamics, Revised Edition. AIAA Education Series. American Institute of Aeronautics and Astronautics, Reston, VA. ISBN: 1-56347-3429., 1999.

Chaturvedi, N., McClamroch, N., and Bernstein, D. Asymptotic smooth stabilization of the inverted 3-d pendulum. IEEE Trans. on Automatic Control, 2009. 54(6):1204-1215. doi:10.1109/TAC.2009.2019792.

Clohessy, W. H. and Wiltshire, R. S. Terminal guidance system for satellite rendezvous. Journal of Aerospace Sciences, 1960. 27(9):653-658.

Egeland, O. and Gravdahl, J. T. Modeling and Simulation for Automatic Control. Marine Cybernetics, Trondheim, Norway. ISBN: 82-92356-01-0., 2002.

Grøtli, E. I. Robust stability and control of spacecraft formations. Ph.D. thesis, Dept. Engineering Cybernetics, Norwegian University of Science \& Technology, 2010.

Hill, G. W. Researches in the lunar theory. American Journal of Mathematics, 1878. 1(1):5-26.

Kapila, V., Sparks, A. G., Buffington, J., and Yan, Q. Spacecraft formation flying: Dynamics and control. In Proc. American Control Conf. San Diego, CA, USA, 1999. doi:10.1109/ACC.1999.786328.
Kelly, R., Santibáñez, V., and Loria, A. Control of robot manipulators in joint space. Advanced textbooks in control engineering. Springer Verlag. ISBN: 1-85233-994-2., 2005.

Kristiansen, R. Dynamic Synchronization of Spacecraft - Modeling and Coordinated Control of LeaderFollower Spacecraft Formations. Ph.D. thesis, Dept. Engineering Cybernetics, Norwegian University of Science \& Technology, 2008.

Kristiansen, R. and Nicklasson, P. J. Spacecraft formation flying: A review and new results on state feedback control. Acta Astronautica, 2009. 65(1112):1537-1552. doi:10.1016/j.actaastro.2009.04.014.

Manikonda, V., Arambel, P. O., Gopinathan, M., Mehra, R. K., and Hadaegh, F. Y. A model predictive control-based approach for spacecraft formation keeping and attitude control. In Proc. American Control Conf. San Diego, CA, USA, 1999. doi:10.1109/ACC.1999.786367.

McInnes, C. R. Autonomous ring formation for a planar constellation of satellites. AIAA Journal of Guidance, Control and Dynamics, 1995. 18(5):12151217.

Ortega, R., Loría, A., and Kelly, R. A semiglobally stable output feedback $\mathrm{PI}^{2} \mathrm{D}$ regulator for robot manipulators. IEEE Trans. on Automatic Control, 1995. 40(8):1432-1436. doi:10.1109/9.402235.

Paden, B. and Panja, R. Globally asymptotically stable 'PD+' controller for robot manipulators. Intl. Journal of Control, 1988. 47(6):1697-1712.

Ploen, S. R., Scharf, D. P., Hadaegh, F. Y., and Acikmese, A. B. Dynamics of Earth orbiting formations. In Proc. AIAA Guidance, Navigation and Control. Providence, RI, USA, 2004.

Scharf, D. P., Hadaegh, F. Y., and Ploen, S. R. A survey of spacecraft formation flying guidance and control (part ii): Control. In Proc. American Control Conf. Boston, MA, USA, 2004.

Schaub, H. and Junkins, J. L. Analytical Mechanics of Space Systems. AIAA Education Series. American Institute of Aeronautics and Astronautics. ISBN: 156347-563-4., 2003.

Schlanbusch, R., Loría, A., Kristiansen, R., and Nicklasson, P. J. PD+ attitude control of rigid bodies with improved performance. In Proc. 49th IEEE Conf. on Decision and Control. Atlanta, GA, USA, 2010. doi:10.1109/CDC.2010.5717227. 
Slotine, J. J.-E. and Li, W. On the adaptive control of robot manipulators. Intl. Journal of Robotics Research, 1987. 6(3):49-59.

Wang, P. K. C. and Hadaegh, F. Y. Coordination and control of multiple microspacecraft moving in formation. Journal of the Astronautical Sciences, 1996. 44(3):315-355.

Yan, Q., Kapila, V., and Sparks, A. G. Pulse-based periodic control for spacecraft formation flying. In Proc. American Control Conf. Chicago, IL, USA, 2000a. doi:10.1109/ACC.2000.878922.

Yan, Q., Yang, G., Kapila, V., and de Queiroz, M. Nonlinear dynamics, trajectory generation, and adaptive control of multiple spacecraft in periodic relative orbits. In Proc. AAS Guidance and Control Conf. Breckenridge, CO, USA, 2000b. 